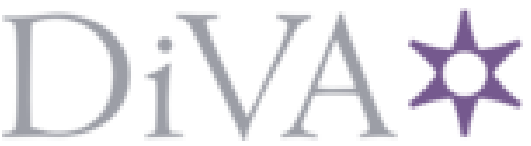

http://www.diva-portal.org

Preprint

This is the submitted version of a paper presented at 4th IEEE International Workshop on Measurement and Network (M\&N), Naples, Italy, Sept. 2017. .

Citation for the original published paper:

Ferrari, P., Flammini, A., Rizzi, M., Sisinni, E., Gidlund, M. (2017)

On the evaluation of LoRaWAN virtual channels orthogonality for dense distributed systems.

In: 4th IEEE International Workshop on Measurement and Network (M\&N)

N.B. When citing this work, cite the original published paper.

Permanent link to this version:

http://urn.kb.se/resolve?urn=urn:nbn:se:miun:diva-3136o 


\section{On the evaluation of LoRaWAN virtual channels orthogonality for dense distributed systems}

\author{
P. Ferrari, A. Flammini, M. Rizzi, E. Sisinni \\ Dept. of Information Engineering \\ University of Brescia \\ Brescia, Italy \\ emiliano.sisinni@unibs.it
}

\author{
M. Gidlund \\ Department of Information Systems and Technology, \\ MidSweden University, \\ Sundsvall, Sweden \\ mikael.gidlund@miun.se
}

\begin{abstract}
Internet of Things (IoT) aims at collecting data from billions of devices connected altogether. Despite there is no one technology able to cope with all possible scenarios, LPWAN solutions are emerging as viable technologies for implementing private, low-cost cellular like wireless networks. Distributed systems could leverage this approach as a driving technology for services as smart environment sensing, pervasive sensing and so on. In the considered scenario, the capacity of the network is of main importance; even if communication is sporadic for most of the time, an event observed by many nodes results in a huge amount of simultaneous transmissions. Are the IoT technologies usable to this end? In this paper LoRaWAN technology is investigated, with the aim of evaluating the orthogonality of virtual channels permitted by the LoRa physical layer. In particular, measurements demonstrated that overlapping transmissions having the same power at the receiver can be correctly decoded if occurring with different spreading factors, whereas co-spread messages require at least $4 \mathrm{~ms}$ spacing.
\end{abstract}

Keywords-LoRaWAN; distributed measurements; IoT; LPWAN; CSS

\section{INTRODUCTION}

Internet of Things (IoT) and smart objects are finding applications in very different domains, ranging from healthcare to industry [1]-[3]. Cloud based solutions are paving the way to innovative services [4]. However, no matter the field, the main challenge to solve is the availability of lowpower and reliable wireless communications [5][6]. In the recent past, the interest towards the so-called Low Power Wide Area Networks (LPWAN) gained unprecedented momentum. These technologies attract commercial interests thanks to the promise of reliable, low-cost cellular-like infrastructure, which could allow for new services and business models. There are many proposals that can be grouped under the LPWAN umbrella; interesting to notice, some of them are managed by consortia that are simultaneously pursuing the standardization process and pushing the commercial deployment in real-world applications. For obvious reasons, most if not all the LPWAN networks operate in the sub-GHz unlicensed bands at 169 , 433, 868/915 MHz. Despite these bands are differently regulated on a regional basis, they are preferred with respect to the worldwide available $2.4 \mathrm{GHz}$ band, which is crowded and suffers from lower penetration capability and shorter range.
Some of the most well accepted LPWANs are SigFox, LoRaWAN and Ingenu [7].

In this paper, authors focus on the LoRaWAN (Long Range WAN), which is considered among the most effective wide-area IoT technologies. Managed by the LoRa Alliance, which also handles the standard regulating the upper layers of the protocol stack, it exploits a proprietary physical layer (the LoRa PHY) originally proposed by Semtech. The LoRaWAN success is mainly imputable to the LoRa innovative chirp modulation technology that permits long-range communication complemented by low power consumption and low cost design. Additionally, its adaptive data rate capability also counteracts interference, providing a tradeoff between the throughput and the robustness. Accordingly, it seems to be an ideal candidate for implementing low-cost, effective distributed measurement systems for smart environment sensing, where things (i.e. sensors) send information (i.e. measurements) to processing and visualizing devices. The applicability in process industry has already been tested by authors [8], as well. However, it is a matter of fact that very few works about network capacity and scalability are available in literature.

On one side, LoRaWAN attracted a lot of developers, thus speeding up its market adoption as well. On the other, most of documents public available are white papers promoted by the same manufacturers. In these documents, "virtual channels" offered by chirp spread spectrum modulation are addressed as the solution for increasing the network capacity. However, as defined in [9], transmissions having a similar spread are susceptible to a new type of interference termed co-spreading factor interference, while transmissions with different spread suffer from inter-spreading factor (or isolation). Consequently, when used as a distributed measurement solutions in a densely populated environment, LoRaWAN networks will be interference-limited, rather than noise-limited.

In this work, a real-world setup, operating according to European regulations, has been arranged in order to evaluate interference-related performance of LoRaWANs. In particular, the orthogonality of "virtual channels" has been experimentally estimated. Paper structure is as follows. In the next section, an overview of the $868 \mathrm{MHz}$ unlicensed band is given. In section III, the basic fundamentals of LoRa and LoRaWAN are provided and in section IV results are 
described. Finally, some conclusions are drawn.

\section{THE $868 \mathrm{MHZ}$ BAND}

The multiple unlicensed bands defined by the European Union (as specified in the ERC Recommendation 70-03), are regulated by the European Telecommunications Standards Institute (ETSI) with the EN300220-1. In this work, the focus is on the $863.0-870.0 \mathrm{MHz}$ region of the spectrum, usually referred to as the $868 \mathrm{MHz}$ band. Any device can utilize this band, as long as it obeys to limitations in terms of the used bandwidth, the Effective Radiated Power (ERP), and the spectrum access method. Regarding the latter point, accessing the $868 \mathrm{MHz}$ band leverages either duty cycling or ListenBefore-Talk (LBT) techniques [10]. The duty cycle $d$ is computed over one hour observation and specifies the maximum accumulated time one device can use for transmitting. Differently said, if $d=1 \%$, any node can use no more of $36 \mathrm{~s}$ per hour for sending data over the air. Conversely, the LBT method obliges the node who wants to transmit to perform a clear channel assessment. In other words, the node has to listen at the selected channel for at least $5 \mathrm{~ms}$ and the transmission can only start if the channel is found not occupied, otherwise the node has to retry later. Furthermore, the LBT also imposes maximum transmit time limits, set to $1 \mathrm{~s}$ for a single transmission and $4 \mathrm{~s}$ for a dialog or polling sequence. The LBT transmitter must also remain silent for at least $100 \mathrm{~ms}$ after a transmission is occurred. Additionally, the total accumulated time of transmission should not exceed $100 \mathrm{~s}$ per hour. The LBT technique can be complemented by Adaptive Frequency Agility (AFA), which means the device can switch to a different channel and thus overall longer transmission times are permitted.

Due to the good propagation characteristics, the $868 \mathrm{MHz}$ band is adopted by several different kind of devices. Consequently, it is split into three regions. The lower portion $(863-865 \mathrm{MHz})$ is designated for wireless microphones, the middle portion $(865-868 \mathrm{MHz})$ is designated for RFID devices, and the remaining portion $(868-870 \mathrm{MHz})$ is designated for more general types of communication. The LoRaWAN devices operate in the latter region, which is further divided into multiple sub-bands, as listed in TABLE I.

TABLE I LORAWAN FREQUENCY PLAN IN AGREEMENT WITH ERC RECCOMMENDATION 70-03 (AS FEBRUARY 2017)

\begin{tabular}{|l|l|l|l|l|}
\hline $\begin{array}{l}\text { Sub- } \\
\text { band }\end{array}$ & $\begin{array}{l}\text { Frequency } \\
\text { Range }[\mathbf{M H z}]\end{array}$ & $\begin{array}{l}\boldsymbol{P}_{\text {MAX,ERP }} \\
{[\mathbf{d B m}]}\end{array}$ & Spectrum Access & $\begin{array}{l}\# \\
\text { LoRa } \\
\text { Ch }\end{array}$ \\
\hline h1.4 & $868.00-868.60$ & 14 & LBT+AFA $d \leq 1 \%$ & 3 \\
\hline h1.5 & $868.70-869.20$ & 14 & LBT+AFA $d \leq 0.1 \%$ & 2 \\
\hline h1.6 & $869.40-869.65$ & 27 & LBT+AFA $d \leq 10 \%$ & 1 \\
\hline h1.7 & $869.70-870.00$ & 14 & LBT+AFA $d \leq 1 \%$ & 1 \\
\hline h1.7 & $869.70-870.00$ & 7 & $d \leq 100 \%$ & 1 \\
\hline
\end{tabular}

As described in the next section, LoRaWAN channel are generally $125 \mathrm{kHz}$ wide but, considering some guard bandwidth, at least $200 \mathrm{kHz}$ are needed. The number of possible LoRaWAN channels is reported in the last column of TABLE I. Interesting to notice, the LoRaWAN specs allow to modify channel to be used dynamically (i.e. time by time) but oblige any device to implement the first three channels, having central frequency $868.1,868.3$, and $868.5 \mathrm{MHz}$, in order to guarantee a minimal common set (e.g. for the preliminary joining procedure).

Other standards different from LoRaWAN operate in the same region. As an example, consider SigFox, some of the Personal Area Network physical layers in the IEEE 802.15.4 standard, the Z-Wave (designed for home-automation) and the Wireless-Mbus (used for smart metering applications). As a consequence, due to the potentially very large area covered with the (high) maximum transmitting power (shown in the third column of TABLE I), coexistence issues could arise as well. Additionally, differently from $2.4 \mathrm{GHz}$ ISM band, where issues are imputable only to intentional radiators (i.e. interferences from other communication systems), the lower the frequency the higher the number of possible unintentional radiators (i.e. unintentional radiators).

\section{LORA AND LORAWAN BASICS}

This section provides an overview of both the physical layer (PHY, i.e. LoRa) and the data link layer (DL, i.e. LoRaWAN). Some details of the overall architecture are resumed as well. Interesting to note, despite the LoRaWAN dates back to 2015 , the academic world only recently started paying attention to it [11][12], mainly for evaluating propagation [13] and security aspects [14]-[16].

\section{A. The physical layer}

As previously stated, the LoRa physical layer leverages Chirp Spread Spectrum (CSS) modulation. Instead of using spreading codes having good correlation properties, each transmitted symbol is coded by a fixed duration linear frequency modulated signal (i.e. a chirp). As a consequence, the larger the time-frequency occupation, the better the interferences immunity.

In regular Binary Orthogonal Keying, where upchirp and downchirp are used for coding ' 1 ' and ' 0 ', or vice versa, spreading is not possible. On the contrary, the LoRa solution maintains constant the chirp duration and modifies the chirp frequency trajectory as a function of the corresponding symbol. In particular, each symbol is represented by the cyclic rotation of a reference chirp, so that different symbols have a sharp edge at different time-frequency locations. The number of bit per symbol is a tunable parameter called the Spreading Factor SF, i.e. only a discrete number $\mathrm{M}=2^{\mathrm{SF}}$ of possible edge locations exists. It can be proved [18] that the effect of shifting in time the reference chirp makes a harmonic to appear in the baseband chirp spectrum, whose frequency depends on the shift amount. Thus, after down-mixing and optimal filtering (i.e. dechirping by correlating with a local copy of the reference chirp) at the receiver, the transmitted symbol can be easily retrieved performing spectral analysis, provided that the phase of each symbol (i.e. the shifted chirp) is continuous and assumes the same value at the beginning and at the end of the symbol itself. Differently said, the phase of the signal has to 
be continuous across symbol boundaries, a feature addressed as inter-symbol phase continuity.

According to European regional regulation of LoRaWAN, the chirp bandwidth is in the range $B \in[125,250] \mathrm{kHz}$, whereas the spreading factor can vary in the range $\mathrm{SF} \in[7 . .12]$ and the chirp duration is $\mathrm{T}_{\mathrm{C}}=2^{\mathrm{SF}} / \mathrm{B}$ (i.e. $\mathrm{B}$ is not affected by the SF). Consequently, the raw bit rate $R_{b}$ at the PHY level is a function of SF as shown in (1). The CR term takes into account possible forward error correction strategy applied to every nibble as an Hamming code with coding rate $\mathrm{CR}=\mathrm{N} / \mathrm{M}$, where $\mathrm{M}=\{5, . ., 8\}$ is the codeword length and $\mathrm{N}=4$ is the data block length; LoRaWAN specs do not tell anything explicitly about CR, but usually commercially available solutions adopts $\mathrm{CR}=4 / 5$. Consequently, adaptive data rate strategies can be implemented where data rate ranges from $\mathrm{DR}=0$ (360bps with $\mathrm{CR}=4 / 5, \mathrm{SF}=12, \mathrm{~B}=125 \mathrm{kHz})$ to $\mathrm{DR}=6$ (11 kbps with $\mathrm{CR}=4 / 5, \mathrm{SF}=7, \mathrm{~B}=250 \mathrm{kHz}$ ); the longer the $\mathrm{SF}$, the lower the bit rate but the more robust the coding.

$$
\mathrm{R}_{\mathrm{b}}=\mathrm{SF} \cdot \frac{\mathrm{B}}{2^{\mathrm{SF}}} \cdot \mathrm{CR}
$$

As stated in the introduction, chirps having different SF are not orthogonal [17], but offer some form of isolation. Differently said, frames transmitted with $\mathrm{SF}=\mathrm{i}$ and $\mathrm{SF}=\mathrm{j}$ can be correctly decoded when overlaps in time and frequency, as long as $\mathrm{i} \neq \mathrm{j}$ and the received packet's Signal to Interference plus Noise Ratio (SINR) is above the isolation threshold (a function of $i$ and $j$ ). As reported in [18], increasing by one the SF provides about $3 \mathrm{~dB}$ of processing gain.

In Fig. 1 it is shown the simultaneous transmission of two messages having the same payload but coded using $\mathrm{SF}=7$ in the $868.1 \mathrm{MHz}$ channel and $\mathrm{SF}=8$ in the $868.3 \mathrm{MHz}$ channel, as acquired by a real-time spectrum analyzer RSA3408A. It is evident that the bandwidth is not affected by the SF, whereas the higher the SF, the longer the chirp duration.

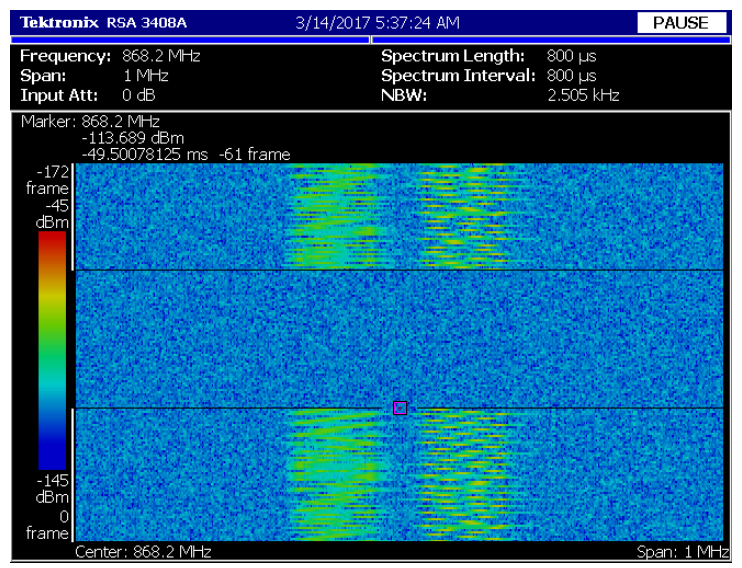

Fig. 1. Acquisition by means of a RSA3408A of two simultaneous LoRaWAN messages in adjacent channels.

The LoRa frame contains a preamble made of eight consecutive unshifted reference chirps for synchronizing the receiver with the transmitter. The frame beginning is marked by the Start of Frame Delimiter (SFD), followed by the PHY
Header. The trailer consists of the PHY-payload Cyclic Redundancy Code (CRC), that is used for uplinks but it is typically not present in downlink frames. It has to be highlighted that, being the radio a proprietary solution, the LoRaWAN specs do not disclose anything about these fields.

\section{B. The data link layer}

LoRaWAN specifications implement the plain ALOHA technique for accessing the medium, but clear channel assessment is theoretically allowed [19]. As described in the previous sub-section, different SFs are pseudo-orthogonal, so that the maximum overall network capacity can be modeled as the superposition of as many independent ALOHA-based networks as the SFs are. Depending on the region of operation, additional limitations deriving from duty-cycle restrictions can apply, as discussed in section II for Europe.

The message structure at the DL level is described in the following and it is shown in Fig. 2.

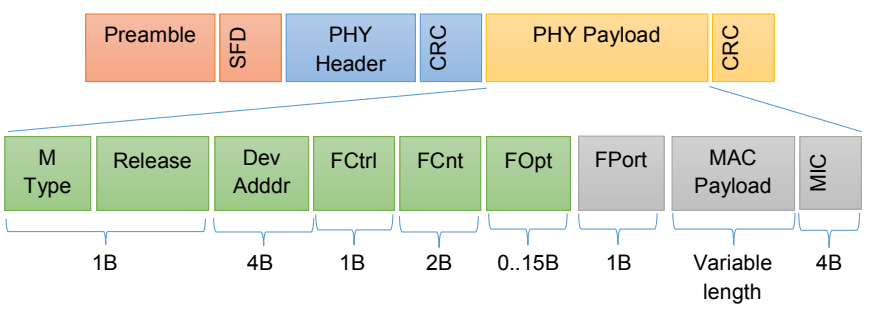

Fig. 2. The LoRaWAN message fields.

The PHY layer payload embeds the MAC layer header, whose fields allow to specify:

- MType, the message type, indicating among other things whether it is an uplink or a downlink message and whether or not it is a confirmed message.

- Release, the LoRaWAN release version.

The subsequent Frame Header contains:

- DevAddr, the (short, 4B) address of the device;

- FCtrl, a control field for specifying if ADR is active or not, if ACK is required or not and the length of The FOpt optional field;

- FCnt, a frame counter to keep aligned transmitter and receiver sides;

- FOpt, an optional field that can transport MAC level commands.

The actual DL payload starts with the FPort, a multiplexing port field (if zero, it means that the payload contains only MAC commands and FOpt has to be absent) and continues with the variable length user data. The MIC, a cryptographic message integrity code, is added for authentication.

As depicted in Fig. 2, the MAC ancillary fields occupy from $8 \mathrm{~B}$ to $23 \mathrm{~B}$ depending on the FOpt field size. In order to maintain the clock drift between the transmitter and the 
receiver reasonable, the maximum MAC level payload length ranges from $242 \mathrm{~B}$ at SF7 down to $51 \mathrm{~B}$ at SF12. If needed, message can be configured as "confirmed", requiring that an acknowledge is sent for finalizing the transaction.

\section{The LoRaWAN architecture}

LoRaWAN architecture resembles a cellular network, as shown in Fig. 3 (i.e. a star topology), as typically happens for IoT solutions. The application layer is not defined at all, leaving the user the freedom to implement its own protocol. The base station is represented by a "packet forwarder", which is nothing but a "gateway" tunneling LoRaWAN messages into the backhaul network and vice versa. No other action is taken by the packet forwarder, since node identification and join procedures are managed by servers in the backend. In this way, the architecture is kept as simple as possible. The protocol stack of the backhaul is out of the LoRaWAN scope. However, in commercially available solutions the backhaul is the Internet or at least an Intranet. The packet forwarder encodes incoming messages into a JSON object transported by an UDP/IP packet toward one or more Network Server(s). The Network Server authenticates the received message and forwards the user payload to a single (or several) Application Server(s) by means of another JSON object transported by a TCP/IP packet. Finally, the Application Server admits nodes to the network and takes care of encrypting/decrypting user data sent/received to/from the end device. An additional Customer Server, receiving JSON-based TCP/IP messages from the Application Server, may exist, allowing a personalized final user application.

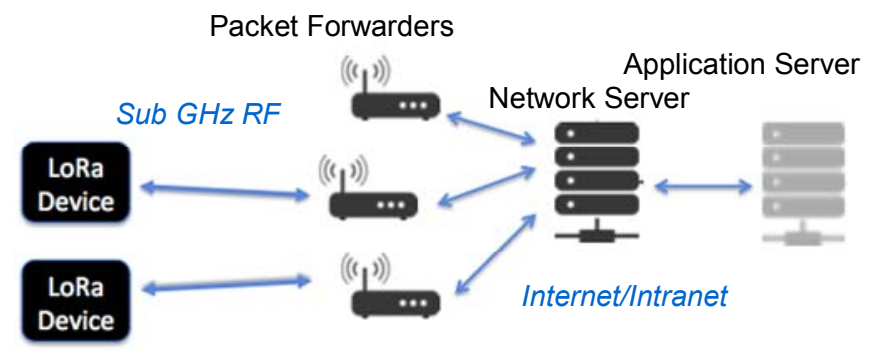

Fig. 3. The LoRaWAN architecture.

\section{EXPERIMENTAL RESULTS}

As stated in the introduction, the aim of the experimental tests is to verify the capability of the LoRaWAN solution to create virtual channels using different SFs. This feature greatly affects network capacity, which is of vital importance in dense environments as very large distributed measurement systems or smart environment sensing applications. It has to be highlighted that

\section{A. Hardware setup}

The experimental setup, depicted in Fig. 4, is implemented using the following components and devices:

- an Agilent 53230A Time interval Counter (TIC), with internal Ovenized Crystal Oscillator (OCXO) time-base option;
- an Agilent 33220A function generator;

- three SX1272 mbed boards, hosting the Semtech's SX1272 LoRa modem, each one interfaced with a STM32 Nucleo board;

- a Multitech LoRa packet forwarder.

A personal computer (PC) is connected to the packet forwarder through an Ethernet link and implements Network and Application Servers functionalities.

It has to be noted that the Multitech packet forwarder uses a SX1301 transceiver, able to simultaneously decode up to 10 channels (one for a FSK communication, one for a LoRaWAN link using a fixed SF and the remaining eight for LoRa messages possibly using any of the available SFs), whereas nodes use a SX1272 transceiver, able to decode only a single channel at a time.

The 53230A acts as the time (and frequency) reference for all the experiments. It has an internal reference output (running at $10 \mathrm{MHz}$ ) used to syntonize the 33220A. A TIC measures the time elapsing between a START and a STOP event, using its internal time-base. This feature has been used to control transmission latency between the 1PPS edge and the "transmission done" signal provided by the SX1272. The 53230A has a single-shot resolution better than 20 ps. The internal time-base (exploiting an OCXO) has a linear aging of $\pm 0.3 \mathrm{ppb}$ in 24 hours. The TIC finite resolution results in $\operatorname{ADEV}(\tau)=2 \times 10^{-11} / \tau$ (from the datasheet).

The function generator is used to generate a 1-pulse-persecond (1PPS) synchronization signal fed to the SX1272 boards. The function generator outputs a rectangular square wave 1PPS signal, triggering the transmission of a new LoRaWAN message. All the nodes are located symmetrically along a circumference whose center is represented by the packet forwarder and whose radius is on the order of $3 \mathrm{~m}$, in order to ensure good signal to noise ratio.

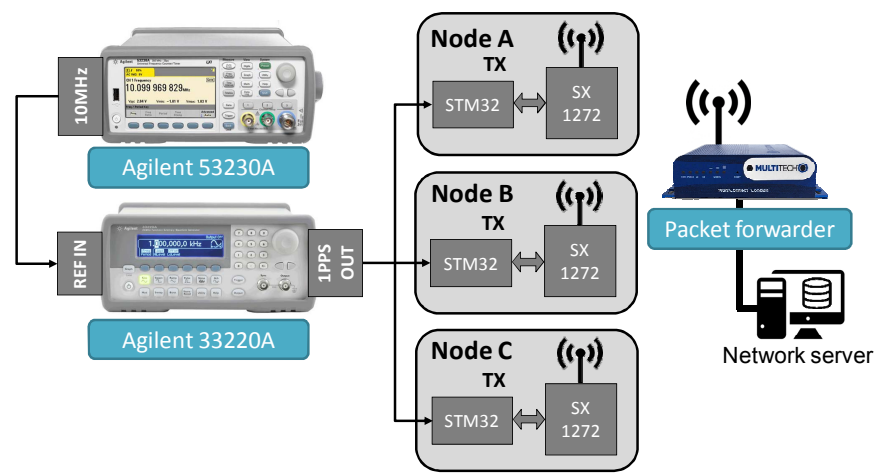

Fig. 4. Experimental setup for the evaluation of the inter-frame spacing in LoRa radio sharing the same channel.

As previously said, SFs are pseudo-orthogonal and should coexist even if frames are overlapped. The scope of the testbed is to precisely schedule message transmission of the boards using the channel allocation described in Fig. 5. Each node is programmed to send a frame of 64 byte.

In detail, in the Fig. 5 (a) and (b) configuration nodes start transmitting simultaneously, whereas in (c) the delay between 
transmissions ("inter-frame spacing") is programmable with a $1 \mathrm{~ms}$ granularity. In particular, rising and falling edge of the 1PPS signal are used for activating transmission on the two different nodes and the 1PPS signal duty cycle is changed accordingly.

(a)

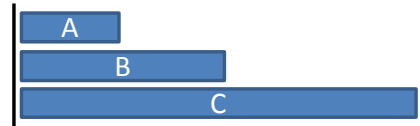

\section{$868.1 \mathrm{MHz}$}

(b)

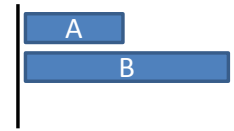

(c)

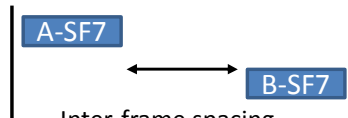

Inter-frame spacing is awakening, it takes more than $120 \mathrm{~ms}$ to send a frame with an overall time-on-air of $118 \mathrm{~ms}$ (the duration of the message using SF7), according to measurements related to SX1272 transceiver hardware signals. The additional time is used to turn on the radio, locking the PLL to the LO, etc and could reflect uncertainty in managing the start of transmission by the radio. Consequently, the behavior of the PER with short interframe delay is probably related to both RF leakage during the awakening stage and possible overlapping of node A message with node B preamble [20][21]. In conclusion, the case (c) results provide useful information for developing a timeslotted approach taking into account that, using the commercially available radios, a time-separation of at least $4 \mathrm{~ms}$ is highly recommended to achieve acceptable PER.

TABLE II RESULTS OF THE COEXISTENCE TEST FOR DIFFERENT SPREADING FACTORS (SF)

\begin{tabular}{|c|c|c|c|c|c|}
\hline Case & Node - SF & $\begin{array}{c}\text { PER } \\
(\%)\end{array}$ & $\begin{array}{c}\text { CRC } \\
\text { Errors }\end{array}$ & $\begin{array}{l}\text { Missed } \\
\text { frames }\end{array}$ & $\begin{array}{l}\text { RSSI } \\
\text { (dBm) }\end{array}$ \\
\hline \multirow{3}{*}{ (a) } & A - SF7 & 1.0 & 9 & 1 & -33 \\
\hline & B - SF8 & 1.5 & 15 & 0 & -35 \\
\hline & C - SF9 & 0.3 & 3 & 0 & -34 \\
\hline \multirow{3}{*}{ (a) } & A - SF8 & 0.5 & 5 & 0 & -33 \\
\hline & B - SF9 & 0.3 & 3 & 0 & -34 \\
\hline & C -SF10 & 0.1 & 1 & 0 & -35 \\
\hline \multirow{3}{*}{ (a) } & A - SF9 & 0.1 & 1 & 0 & -33 \\
\hline & B - SF10 & $<0.1$ & 0 & 0 & -31 \\
\hline & $\mathrm{C}-\mathrm{SF} 11$ & $<0.1$ & 0 & 0 & -33 \\
\hline \multirow{3}{*}{ (a) } & $\mathrm{A}-\mathrm{SF} 10$ & $<0.1$ & 0 & 0 & -31 \\
\hline & B - SF11 & $<0.1$ & 0 & 0 & -32 \\
\hline & $\mathrm{C}-\mathrm{SF} 12$ & $<0.1$ & 0 & 0 & -33 \\
\hline \multirow{2}{*}{ (b) } & A - SF7 & 1.0 & 10 & 0 & -32 \\
\hline & B - SF8 & 1.6 & 16 & 0 & -33 \\
\hline \multirow{2}{*}{ (b) } & $\mathrm{A}-\mathrm{SF} 8$ & 0.3 & 3 & 0 & -34 \\
\hline & B - SF9 & 0.4 & 4 & 0 & -33 \\
\hline \multirow{2}{*}{ (b) } & A-SF7 & 1.2 & 12 & 0 & -33 \\
\hline & B-SF9 & 0.6 & 6 & 0 & -33 \\
\hline
\end{tabular}

Thanks to obtained result, it would be possible to implement simple simulation models of LoRaWAN networks, e.g. expanding results found in [21], which provided results after evaluation based on a "over-the-cable" setup (using devices similar to those adopted in this work). As an example, a very rough simulation model could be the one in Fig. 7.

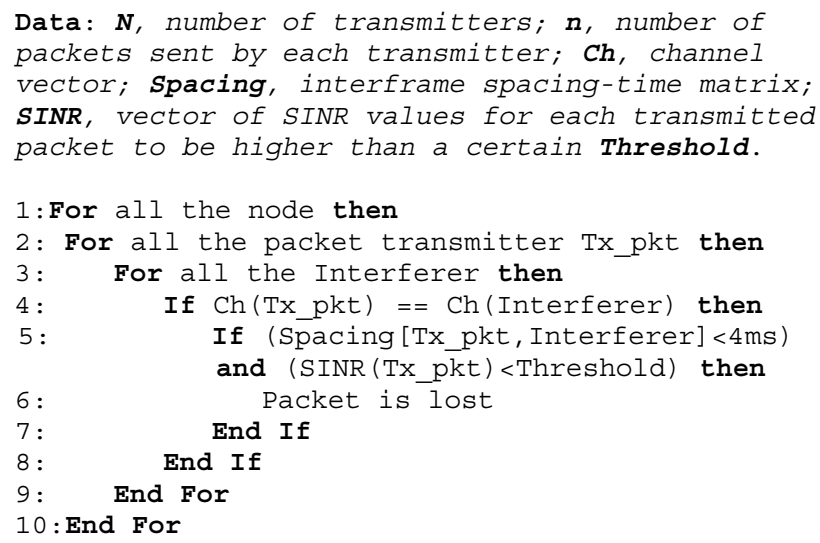

node B is consistently below the PER of node A for an interframe delay equal and above zero ms. With an inter-frame delay higher than zero $\mathrm{ms}$, the expected result is an equal PER for both nodes, however a slightly higher PER of node A (dominated by CRC errors) has been detected in the $0-4 \mathrm{~ms}$ region. It has been experimentally evaluated that when a node
Fig. 7. Simple model for determining packet losses according to case (c) experimental results. 
The considered scenario is based on $\mathrm{N}=1000$ nodes connected to a single packet forwarder. All of them transmit their packets "as soon as possible" on a randomly chosen channel in the h1.4 sub-band respecting the duty-cycle limitation and using the SF7. Start of transmission for each packet is delayed by $\Delta$, uniformly distributed in the range $\Delta \in[0, \tau)$ where $\tau$ is the packet duration. For sake of simplicity, packets at the receiver have SINR values above and below the Threshold according to a uniform distribution. If ten simulation iterations are run, the average packet loss varies from $0.42 \%$ for $20 \mathrm{~B}$-long packets, down to $0.33 \%$ for $64 \mathrm{~B}-$ long packet. The result is in accordance with the fact that the longer is the packet, the fewer are the number of packets allowed if duty-cycle constrains are respected.

\section{CONCLUSIONS}

In this work the LoRaWAN solution, an example of LPWAN solution for IoT applications, has been experimentally evaluated in order to assess the behavior in dense environments. Despite it adopts the ALOHA medium access strategy, a relatively large number of nodes can be reached using virtual channels permitted by the CSS modulation. A purposely designed experimental setup has been created to allow the evaluation of the communication performance in case simultaneous communication occur in the same area. Generally speaking, the orthogonality is verified: if different SFs are used (i.e. PER is satisfactory), otherwise collision may destroy the messages. Nevertheless, it has been experimentally found that a synchronized approach (time slotted) could improve the overall performance: PER can be increase also in the case of identical SF if an inter frame space greater than $4 \mathrm{~ms}$ exists between packets.

\section{ACKNOWLEDGMENT}

The research has been partially funded by research grant MIUR SCN00416, "Brescia Smart Living: Integrated energy and services for the enhancement of the welfare" and by University of Brescia H\&W grant "AQMaSC" and $\mathrm{H} \& \mathrm{~W}$ grant "Smart Break 2".

\section{REFERENCES}

[1] B. Xu, L. D. Xu, H. Cai, C. Xie, J. Hu and F. Bu, "Ubiquitous Data Accessing Method in IoT-Based Information System for Emergency Medical Services" in IEEE Transactions on Industrial Informatics, vol. 10, no. 2, pp. 1578-1586, May 2014.

[2] P. Bellagente, P. Ferrari, A. Flammini, S. Rinaldi, "Adopting IoT framework for Energy Management of Smart Building: A real testcase", Proc. Of IEEE 1st International Forum on Research and Technologies for Society and Industry, RTSI, pp. 138-143.

[3] P. Bellagente, P. Ferrari, A. Flammini, S. Rinaldi, E. Sisinni, "Enabling PROFINET devices to work in IoT: Characterization and requirements", Proc. of IEEE Instrumentation and Measurement Technology Conference, 2016-July.

[4] L. Ascorti, S. Savazzi, G. Soatti, M. Nicoli, E. Sisinni and S. Galimberti, "A Wireless Cloud Network Platform for Industrial Process Automation: Critical Data Publishing and Distributed Sensing", in IEEE Transactions on Instrumentation and Measurement, vol. 66, no. 4, pp. 592-603, April 2017.
[5] A. Autolitano et al., "Wireless for the factory: The Wi-Fact analysis", 2015 IEEE 1st International Forum on Research and Technologies for Society and Industry Leveraging a better tomorrow (RTSI), Turin, 2015, pp. 403-410.

[6] G. Patti, L. Leonardi and L. Lo Bello, "A Bluetooth Low Energy realtime protocol for Industrial Wireless mesh Networks", IECON 2016 42nd Annual Conference of the IEEE Industrial Electronics Society, Florence, 2016, pp. 4627-4632.

[7] U. Raza, P. Kulkarni and M. Sooriyabandara, "Low Power Wide Area Networks: An Overview", in IEEE Communications Surveys \& Tutorials, vol. 19, no. 2, pp. 855-873, Secondquarter 2017.

[8] M. Rizzi, P. Ferrari, A. Flammini, E. Sisinni, M. Gidlund, "Using LoRa for industrial wireless networks", 2017 IEEE 13th International Workshop on Factory Communication Systems (WFCS), Trondheim, Norway, May 31 - June 2, 2017, pp. 1-4

[9] O. Georgiou and U. Raza, "Low Power Wide Area Network Analysis: Can LoRa Scale?" in IEEE Wireless Communications Letters, vol. 6, no. 2, pp. 162-165, April 2017.

[10] M. Lauridsen, B. Vejlgaard, I. Z. Kovacs, H. Nguyen and P. Mogensen, "Interference Measurements in the European $868 \mathrm{MHz}$ ISM Band with Focus on LoRa and SigFox”, 2017 IEEE Wireless Communications and Networking Conference (WCNC), San Francisco, CA, 2017, pp. 1-6.

[11] V. A. Stan, R. S. Timnea and R. A. Gheorghiu, "Overview of high reliable radio data infrastructures for public automation applications: LoRa networks", 2016 8th International Conference on Electronics, Computers and Artificial Intelligence (ECAI), Ploiesti, 2016, pp. 1-4.

[12] A. J. Wixted, P. Kinnaird, H. Larijani, A. Tait, A. Ahmadinia and N. Strachan, "Evaluation of LoRa and LoRaWAN for wireless sensor networks", 2016 IEEE SENSORS, Orlando, FL, 2016, pp. 1-3.

[13] L. Gregora, L. Vojtech and M. Neruda, "Indoor signal propagation of LoRa technology", 2016 17th International Conference on Mechatronics - Mechatronika (ME), Prague, 2016, pp. 1-4.

[14] S. Tomasin, S. Zulian and L. Vangelista, "Security Analysis of LoRaWAN Join Procedure for Internet of Things Networks," 2017 IEEE Wireless Communications and Networking Conference Workshops (WCNCW), San Francisco, CA, 2017, pp. 1-6.

[15] JungWoon Lee, DongYeop Hwang, JiHong Park and Ki-Hyung Kim, "Risk analysis and countermeasure for bit-flipping attack in LoRaWAN," 2017 International Conference on Information Networking (ICOIN), Da Nang, 2017, pp. 549-551.

[16] SeungJae Na, DongYeop Hwang, WoonSeob Shin and Ki-Hyung Kim, "Scenario and countermeasure for replay attack using join request messages in LoRaWAN," 2017 International Conference on Information Networking (ICOIN), Da Nang, 2017, pp. 718-720.

[17] B. Reynders and S. Pollin, "Chirp spread spectrum as a modulation technique for long range communication," 2016 Symposium on Communications and Vehicular Technologies (SCVT), Mons, 2016, pp. $1-5$.

[18] C. Goursaud, J. M. Gorce, "Dedicated networks for IoT: PHY / MAC state of the art and challenges", IOT EAI, DOI: 10.4108/eai.26-102015.150597.

[19] K. Mikhaylov, .. Juha Petaejaejaervi and T. Haenninen, "Analysis of Capacity and Scalability of the LoRa Low Power Wide Area Network Technology" European Wireless 2016; 22th European Wireless Conference, Oulu, Finland, 2016, pp. 1-6.

[20] M. Bor, U. Roedig, T. Voigt, J. Alonso, "Do LoRa Low-Power WideArea Networks Scale?", 19th ACM International Conference on Modeling, Analysis and Simulation of Wireless and Mobile Systems, November 2016.

[21] J. Haxhibeqiri, F. Van den Abeele, I. Moerman, J. Hoebeke, "LoRa Scalability: A Simulation Model Based on Interference Measurements", Sensors 2017, Vol.17, N.6, Page 1193. 